\title{
Publicación múltiple de artículos en Psicología: Definición de producción atípica, probable y normal
}

\section{Publication of multiple articles in psychology: Definition of the atypical, probable and normal production}

\author{
Jéssica Sanmarco ${ }^{1}$, Verónica Marcos $^{2}$, Dolores Seijo ${ }^{3}$, Mercedes Novo ${ }^{4}$ \\ ${ }^{1}$ Unidad de Psicología Forense, Universidad de Santiago de Compostela. jessica.sanmarco.vazquez@usc.es \\ ${ }^{2}$ Unidad de Psicología Forense, Universidad de Santiago de Compostela.veronica.marcos@rai.usc.es \\ ${ }^{3}$ Psicología Organizacional, Jurídica Forense y Metodología de las Ciencias del Comportamiento, Universidad de Santiago de Compostela \\ mariadolores.seijo@usc.es \\ ${ }^{4}$ Psicología Organizacional, Jurídica Forense y Metodología de las Ciencias del Comportamiento, Universidad de Santiago de Compostela \\ mercedes.novo@usc.es
}

Recibido: 24/10/2019

Aceptado: 9/12/2019

Copyright $(\mathrm{C}$

Facultad de CC. de la Educación y Deporte. Universidad de Vigo

\section{Dirección de contacto:}

Mercedes Novo

Facultad de Psicología

Universidad de Santiago de Compostela

15782 SANTIAGO DE COMPOSTELA, A

CORUÑA (ESPAÑA)

\begin{abstract}
Resumen
Se diseñó un estudio de archivo para conocer las tasas de multi-publicación de los investigadores españoles de Psicología en revistas de prestigio. Para ello, se calculó el promedio de artículos publicados por autor y año natural en revistas indexadas en la Core Collection de la Web of Science por 551 investigadores, clasificados por área de conocimiento. Los resultados mostraron que el $5 \%$ de los investigadores tenían una producción atípica (valores outliers o extremos) y, por el contrario, otro $5 \%$ no tenía producción científica. La autoría probable de artículos por autor oscila, según área de conocimiento, entre alrededor de 1 artículo por año (límite inferior del intervalo) y en torno a 2 y 2,5 (límite superior del intervalo) por año, publicando la mitad de la distribución (mediana) más de un artículo al año. El límite superior del intervalo de normalidad establece que el $5 \%$ de los investigadores publican en promedio, según área de conocimiento, ligeramente más de 2 ó 3 artículos al año. Además, los resultados pusieron de manifiesto diferencias significativas en la tasa de multi-publicación entre las áreas de conocimiento, registrándose en el área de Personalidad, Evaluación y Tratamiento Psicológico la mayor tasa de multipublicación por autor. Se discuten las implicaciones para la evaluación de la actividad investigadora del profesorado de Psicología.
\end{abstract}

\section{Palabras clave}

Producción Científica, Evaluación de la Investigación, Multi-publicación, Valores Atípicos, Valores Normales

\begin{abstract}
With the aim of knowing the multi-publication rate of the Spanish researchers in the field of Psychology in prestigious journals, an archival study was designed. As for this, the average of scientific papers published by author and natural year in journals indexed in the Core Collection of the Web of Science by 551 researchers classified by knowledge area was computed. The results showed that $5 \%$ of the researchers had an atypical production (outliers or extreme values) and, on the contrary, other $5 \%$ had
\end{abstract}


no scientific production. The probable authorship of papers by author ranged, according to knowledge area, between around 1 paper per year (lower limit of the interval) and around 2 and 2,5 (upper limit of the interval) by year, publishing the middle of the distribution more than 1 paper per year. The upper limit of the normal interval stated that $5 \%$ of the researchers publish an average, according to the knowledge area, slightly more than 2 or 3 papers per year. Moreover, the results exhibited significant differences among the knowledge areas in the multi-publication rate, registering the highest rates in the area of Clinical Psychology. The implications of the results for the assessment of the research activity of the Psychology researchers are discussed.

\section{Key Words}

Scientific Production, Research Evaluation, Multi-publication, Atypical Values, Probable Values, Normal Values

\section{INTRODUCCIÓN}

En la relación de criterios específicos en la convocatoria de evaluación de la actividad investigadora de la Comisión Nacional Evaluadora de la Actividad Investigadora (CNEAI), ésta fija, para todos los campos, que para que una aportación sea considerada, el solicitante deberá haber participado activamente en los trabajos que le dieron origen, concretando su aportación específica a los mismos en los casos de multiautoría (Resolución de 14 de noviembre de 2018 [Resolución 14/11/2018], de la Comisión Nacional Evaluadora de la Actividad Investigadora, por la que se publican los criterios específicos aprobados para cada uno de los campos de evaluación). Se define en la convocatoria la multiautoría como un elevado (o desproporcionado) número de autores no justificado por la naturaleza y contenidos de la investigación. Y, en cada campo de evaluación, se sintetiza de un modo ligeramente diferente. Para todos los campos, dicha resolución fija que para que una aportación sea considerada, el solicitante deberá haber participado activamente en los trabajos que le dieron origen, concretando su aportación específica a los mismos en los casos de multiautoría. Y en el campo de la Psicología concreta que un número desproporcionado de autores podrá reducir la calificación asignada a una aportación, debiendo estar justificado por el tema, su complejidad y su extensión y el solicitante ha de justificar su contribución a cada una de las aportaciones en coautoría. Ahora bien, esta definición de multiautoría es incompleta debiendo completarse con la producción del autor, esto es, por la multiplicidad de publicaciones. De este modo, un autor puede publicar un número excesivo o desproporcionado de aportaciones, entendiéndose por aportaciones en el campo de la Psicología artículos científicos, y libros o capítulos que sean resultado de investigación (apartados 3.a a 3.e; Resolución 14/11/2018), pero que el criterio orientador para la obtención de una evaluación positiva exige que, al menos, 4 de las aportaciones sean artículos publicados en revistas de prestigio, aceptándose como tales las incluidas en los listados del JCR, tanto de la Social Science Edition como de la Science Edition (apartado 3.a). Sucintamente, a los autores con un número excesivo o desproporcionado de artículos publicados en revistadas indexadas en el JCR, en línea con la creciente demanda de las políticas de ética e integridad en las publicaciones y de calidad de las mismas (Codina, 2019), se les debería solicitar una justificación de la participación en 
las aportaciones, reduciendo, en su caso, la calificación numérica asignada a la(s) aportación(es).

La unidad de medida de la producción de los investigadores es el año natural (no el académico) y el período de evaluación es de 6 años (sexenio) con dedicación a tiempo completo o período equivalente si es a tiempo parcial (Real Decreto 1086/1989, de 28 de agosto, sobre retribuciones del profesorado universitario), siendo el número máximo de aportaciones que se pueden presentar de cinco (Orden de 2 de diciembre de 1994 del Ministerio de Educación y Ciencia). En consecuencia, la normativa relaciona el tiempo de dedicación con la producción científica y se orienta a una evaluación de hasta 5 aportaciones científicas que impliquen un progreso real del conocimiento.

Para conocer la plausibilidad de esta contingencia (autoría excesiva o desproporcionada), llevamos a cabo previamente al estudio en sí un análisis de cohorte de los 50 autores más productivos de Psicología (categoría "Psychology") en número de artículos y revisiones de la literatura (los artículos que son revisiones de la literatura, sistemáticas o meta-analíticas son codificadas como "revisiones") publicados en revistas indexadas en la Core Collection de la Web of Science en los años 1998, 2008 y 2018 (cohortes de 10 años); se advirtieron diferencias en el promedio de artículos publicados por autor, $\mathrm{F}(2,147)=22,88, \mathrm{p}<, 001$. Los contrastes a posteriori (Bonferroni) corroboraron que en $2008(\mathrm{M}=17,78)$ el promedio de artículos firmados por los autores más productivos era significativamente mayor que en $1998(\mathrm{M}=13,08, \mathrm{~d}=0,76)$, y en $2018(M=26,88, d=0,79)$ que en 2008. Resultado muy robusto, $1-\beta=1$. En otros términos, entre 1998 y 2008 la tasa de producción de los autores más productivos subió el $35,5 \%(r=, 355)$ y de 2008 a 2018 , el $36,7 \%(r=, 367)$. En suma, el número de artículos firmados por los autores más productivos se ha venido incrementando con el tiempo significativamente resultando excesivo para una dedicación total a los mismos. Si nos atenemos a los criterios de valoración de la CNEAI en los que se ha de justificar la participación en la aportación, reduciendo la calificación de no ser total, cabe preguntarse cuál es el número de artículos que un autor puede producir por año (unidad de la medida de la convocatoria; Real Decreto 1086/1989).

Veamos un ejemplo concreto, Newhart M. firmó 91 artículos/revisiones en 2018 lo que sale a un promedio de 1 artículo cada 4 días (365 totales en 2018), que bajaría a menos de 3 si se toman los días laborales (249 días). En todo caso, una producción tan prolija como difícil de justificar. En tanto que el autor con menos publicaciones de los 50 más productivos en 2018 firmó 16 artículos lo que supone un artículo cada 23 días (15 en días laborales), cifra que, a tenor de los diseños, recogida y análisis de datos, estudio de la literatura y redacción del artículo, resulta imposible para una aportación al total del artículo en cualquiera de las áreas de conocimiento del campo de la Psicología.

Por todo ello nos planteamos un estudio de archivo para conocer si el crecimiento general en la multiautoría, incluso hasta límites fuera de lo normal (valores atípicos), está relacionado también con la publicación múltiple de artículos de los autores y, en este caso, identificar, por área de conocimiento, los puntos de corte para los valores de producción atípicos, la autoría probable de artículos, los límites inferior y superior de la normalidad en la publicación de artículos, y las probabilidades de los investigadores de poder optar a una evaluación positiva de la investigación (sexenios) en condiciones de poder cumplir con el criterio orientador. 


\section{MÉTODO}

\subsection{Base de datos}

Como método de identificación de investigadores se tomó le listado de la Web of Science Production of Spanish Psychology (http://www.psy-wos.es/). Se identificaron por este medio 551 investigadores productivos en la disciplina de Psicología registrados el 6 de septiembre de 2019. A partir de estos se buscó por el ResearcherID (Publons) cada uno de ellos obteniendo las estadísticas individualizadas. Como el ResearcherID engloba el total de publicaciones (e.g., artículos, revisiones, proceedings paper, meeting abstract, book chapter, book, book review, correction, note, editorial material, letter, discussion, reprint, early access, new item, biographical ítem, bibliography, script, software review), se procedió con el algoritmo de búsqueda "authors identifiers", seleccionando a continuación en "Document types" los registros de "Article" y "Review" (el criterio orientador para la obtención de una evaluación positiva establece que, al menos, 4 de las aportaciones han de ser artículos; Resolución 14/11/2018). Con el resultado de la búsqueda para cada investigador, se creó una base de datos por autor con el número de registros observados por año de publicación. Los investigadores fueron codificados por área de conocimiento en función de la clasificación que obra en la Web of Science Production of Spanish Psychology.

La creación de la base de datos fue replicada por un segundo investigador y sometida a un análisis de concordancia, kappa verdadera $\left({ }^{-} \kappa\right)$, hallándose una concordancia total, es decir, la base de datos para este estudio representa fidedignamente la realidad de la misma (Fariña, Arce y Novo, 2002).

\subsection{Análisis de datos}

El análisis de datos se llevó a cabo con el SPSS y hojas de cálculo creadas por los autores (tamaños del efecto). Primeramente, se llevó a cabo un análisis descriptivo de las variables (i.e., media, error estándar de la media, desviación estándar, mediana, varianza, valor mínimo y valor máximo) y estudio de la normalidad de la variable de medida (asimetría y curtosis). Valores en la asimetría o cursotis entre $>-2$ ó $>+2$ advierten de una distribución anormal de la variable (George y Mallery, 2010). Para la identificación de los valores atípicos se procedió a una exploración de los datos con el objetivo de identificar los autores con valores de producción media de artículos outliers $\left( \pm 1,5^{*} \mathrm{IQR}\right)$ y extremos $( \pm 3 * \mathrm{IQR})$, así como los puntos de corte para la clasificación de éstos por área de conocimiento. Se calculó el intervalo de confianza al 95\% para la proporción de valores atípicos encontrados con el objeto de conocer si entraban (el límite superior del intervalo no alcanzaba ,05), igualaban (el intervalo pasa por ,05) o superaban (el límite inferior del intervalo es mayor que ,05) la probabilidad esperada por azar $(, 05)$.

Eliminados los valores atípicos de las distribuciones y normalizadas (asimetría y curtosis $<-2 \mathrm{y}+2$ ), se calcularon los intervalos de normalidad en la producción de artículos por año $(\mathrm{M} \pm \mathrm{DE} * 1,645)$ y sexenio, y de autoría probable de artículos $\left(\mathrm{M} \pm \mathrm{DE}^{*}, 6745\right)$ por año y sexenio.

La comparación de medias se llevó a cabo mediante un ANOVA con los contrastes a posteriori mediante la prueba T3 de Dunnett, cuando no se observó homogeneidad de 
varianzas y Ns $>30$, y con el nivel de protección de Bonferroni cuando las variables variaban homogéneamente.

La estimación de las tasas de incremento de una media sobre otra se obtuvo transformando d a r (Amado, Arce y Herraiz, 2015).

Para la interpretación de los tamaños del efecto se procedió con la técnica desarrollada por Arce (Monteiro, Vázquez, Seijo y Arce, 2018), y la probabilidad de superioridad del tamaño del efecto (Vilariño, Amado, Vázquez y Arce, 2018).

\section{RESULTADOS}

Se calculó el promedio de artículos firmados por cada autor (artículos indexados en la Core Collection de la Web of Science / número de años de publicación, con el recuento de los años transcurridos desde el primer artículo indexado y 2018). No obstante, los resultados evidenciaron que las distribuciones no son normales (i.e., asimetría o curtosis $>2$ o $<-2$ ), a excepción del área de Psicología Social. La curtosis nos informa que las distribuciones son leptocúrticas (más puntiagudas que una distribución normal), esto es, que hay una concentración excesiva de valores en torno a la media. Y, en el caso de Psicología Básica, además, es asimétrica a la derecha. Por ello, la media y sus índices de dispersión no son robustos. No obstante, la mediana, robusta ante la falta de normalidad, advierte que entre 1,18 (Psicología Social) y 1,49 (Personalidad, Evaluación y Tratamiento Psicológico) artículos firmados por año es el valor que parte la distribución por la mitad. En otras palabras, la mitad de los investigadores firman entre menos de 1,18 y 1,49 artículos por año. También son de destacar los valores mínimos, que sitúan a los investigadores menos productivos en unos niveles de publicación nulos o prácticamente nulos, en tanto los valores máximos indican que los autores más productivos publican en torno a 8 artículos al año en las áreas de Psicobiología; Personalidad, Evaluación y Tratamiento Psicológico; Psicología Básica; y Metodología de las Ciencias de Comportamiento; y a 5 en Psicología Evolutiva y de la Educación, y sobre 4 en Psicología Social.

\begin{tabular}{lcccccc}
\hline Psicobiología & PETRA & $\begin{array}{c}\text { Evolutiva y } \\
\text { Educación }\end{array}$ & Básica & Metodología & Social \\
\hline $\mathbf{N}$ & 76 & 148 & 84 & 92 & 49 & 102 \\
\hline $\mathbf{M}$ & 1,69 & 1,89 & 1,37 & 1,57 & 1,82 & 1,39 \\
\hline EEM & 0,15 & 0,13 & 0,11 & 0,15 & 0,20 & 0,10 \\
\hline Md & 1,26 & 1,49 & 1,25 & 1,22 & 1,47 & 1,18 \\
\hline DE & 1,31 & 1,56 & 0,98 & 1,42 & 1,41 & 1,03 \\
\hline S2 & 1,71 & 2,42 & 0,96 & 2,03 & 1,98 & 1,07 \\
\hline Mín. & 0,00 & 0,09 & 0,09 & 0,08 & 0,20 & 0,08 \\
\hline Máx. & 7,64 & 7,90 & 4,92 & 7,96 & 7,65 & 4,32 \\
\hline As & 1,95 & 1,55 & 1,52 & 2,25 & 1,75 & 0,91 \\
\hline Kur & 5,30 & 2,93 & 2,72 & 6,09 & 4,86 & 0,19 \\
\hline
\end{tabular}

Nota. PETRA: Personalidad, Evaluación y Tratamiento Psicológico; N: Número total de investigadores; M: Media; EEM: Error estándar de la media; Md: Mediana; DE: Desviación estándar; S2: Varianza; Mín.: Valor mínimo; Máx.: Valor Máximo; As: Asimetría; Kur: Curtosis.

Tabla 1. Descriptivos de las distribuciones de autores por área 
Los resultados anteriores llevaron a que se exploraran los valores atípicos (outliers y extremos) en las distribuciones. Los resultados revelaron (ver Tabla 2) la presencia de valores atípicos en todas las áreas de conocimiento, incluso con probabilidades que no entran en los márgenes del azar $(<, 05)$ en las áreas de Psicobiología; Personalidad, Evaluación y Tratamiento Psicológico; Psicología Evolutiva y de la Educación; y Psicología Básica (el intervalo de confianza al 95\% pasa por ,05). De hecho, la contingencia de valores atípicos afecta también al total de Psicología, ,047[,029, ,065], que es igual al límite de presencia de dichos valores al azar (el intervalo de confianza al 95\% para la proporción observada pasa por ,05, el azar). Sólo en el área de Psicología Social, la tasa de valores atípicos es inferior a la esperada por azar (el límite superior del intervalo de confianza es inferior al azar, ,05).

\begin{tabular}{lccccc}
\hline \multicolumn{1}{c}{ Área (N) } & \multicolumn{2}{c}{ Outliers } & \multicolumn{2}{c}{ Extremos } & Total \\
\hline & $\mathrm{PC}$ & $\mathrm{n}$ & $\mathrm{PC}$ & $\mathrm{n}$ & $\mathrm{n}(\mathrm{p}[\mathrm{IC95 \%}])$ \\
\hline Psicobiología (76) & $>3,86$ & 5 & $>5,60$ & 1 & $6(, 078[, 018,, 138])$ \\
\hline PETRA (148) & $>5,57$ & 6 & $>8,49$ & 0 & $6(, 041[, 009,, 072])$ \\
\hline Evolutiva y Educación (84) & $>3,60$ & 4 & $>5,35$ & 0 & $4(, 048[, 002,, 094])$ \\
\hline Metodología (49) & $>5,46$ & 1 & $>8,31$ & 0 & $1(, 020[-, 019,, 059])$ \\
\hline Básica (92) & $>3,72$ & 4 & $>5,56$ & 3 & $7(, 076[, 022,, 130])$ \\
\hline Social (102) & $>3,85$ & 2 & $>5,83$ & 0 & $2(, 020[-, 007,, 047])$ \\
\hline
\end{tabular}

Nota. PC: Punto de Corte; p[IC95\%]: Probabilidad observada [intervalo de confianza para la probabilidad observada al 95\%]; PETRA: Personalidad, Evaluación y Tratamiento Psicológico

Tabla 2. Exploración de valores outliers y extremos en el número de autores por el área de conocimiento.

\begin{tabular}{|c|c|c|c|c|c|c|c|}
\hline Área & As & Kurt & $\mathbf{N}$ & M(IC 95\%) & Md & APA & IN90\% \\
\hline Psicobio. & 0,850 & 0,700 & 70 & $\begin{array}{c}1,41[1,22 \\
1,60]\end{array}$ & $1,77(10,6)$ & $\begin{array}{l}0,85(5,1), \\
1,97(11,8)\end{array}$ & $\begin{array}{l}0,04(0,2), \\
2,78(16,7)\end{array}$ \\
\hline PETRA & 0,705 & $-0,388$ & 142 & $\begin{array}{c}1,68[1,49, \\
1,87]\end{array}$ & $1,41(8,5)$ & $\begin{array}{l}0,89(5,3), \\
2,47(14,8)\end{array}$ & $\begin{array}{c}-0,24(0), \\
3,60(21,6)\end{array}$ \\
\hline Evol. y Edu. & 0,708 & 0,097 & 80 & $\begin{array}{c}1,22[1,06, \\
1,38]\end{array}$ & $1,22(7,3)$ & $\begin{array}{l}0,73(4,4), \\
1,71(10,3)\end{array}$ & $\begin{array}{l}0,04(0,2) \\
2,40(14,4)\end{array}$ \\
\hline Básica & 1,001 & 0,700 & 85 & $\begin{array}{c}1,25[1,08, \\
1,42]\end{array}$ & $1,20(7,2)$ & $\begin{array}{l}0,70(4,2) \\
1,80(10,8)\end{array}$ & $\begin{array}{r}-0,08(0), \\
2,58(15,5)\end{array}$ \\
\hline Metodología & 0,723 & $-0,325$ & 48 & $\begin{array}{c}1,70[1,39 \\
2,01]\end{array}$ & $1,46(8,8)$ & $\begin{array}{l}0,94(5,6) \\
2,46(14,8)\end{array}$ & $\begin{array}{r}-0,16(0), \\
3,56(21,4)\end{array}$ \\
\hline Social & 0,822 & $-0,018$ & 100 & $\begin{array}{c}1,34[1,15, \\
1,53]\end{array}$ & $1,16(7,0)$ & $\begin{array}{l}0,69(4,1) \\
1,99(11,9)\end{array}$ & $\begin{array}{c}-0,24(0) \\
2,92(17,5)\end{array}$ \\
\hline
\end{tabular}

Nota. As: Asimetría; Kurt: Curtosis; N: Número total de investigadores; M(IC 95\%): Media (Intervalo de Confianza para la Media al 95\%); Md: Mediana (artículos por sexenio); APA: Autoría Probable de Artículos (artículos por sexenio); IN90\%: Intervalo de Normalidad al 90\% (artículos por sexenio); PETRA: Personalidad, Evaluación y Tratamiento Psicológico

Tabla 3. Valores críticos y puntos de corte para la identificación de un número excesivamente bajo y alto de artículos publicados por autor y año

El promedio de artículos firmado por autor y área por año natural (ver Tabla 3) oscila entre 1,22 (Psicología Evolutiva y de la Educación) y 1,70 (Metodología de las Ciencias del Comportamiento). Por su parte, el intervalo de confianza para la media, que no se solapa entre todos ellos, lo que abre la puerta al estudio de la comparación de medias, abarca entre alrededor de 1 (Psicología Evolutiva y de la Educación, y Psicología Básica) a un máximo de 2 (Metodología). La mediana viene a confirmar que la mitad de los investigadores publican menos de 2 artículos por año: como máximo 1,77 en 
Psicobiología; en torno a 1,5 en Metodología de las Ciencias del Comportamiento; y cerca de 1 en Psicología Social; Psicología Básica; y Personalidad, Evaluación y Tratamiento Psicológico. A su vez, la autoría probable, esto es, el 50\% central de la distribución, identifica en todas las áreas como límite inferior menos de un artículo, esto es, entre 4 y 5 artículos cada 6 años (sexenio), y el superior entre en torno a 10 y 15 cada 6 años. Finalmente, la normalidad (intervalo de normalidad) comprende entre un límite inferior de ningún artículo publicado al año, es decir, al menos el 5\% de los investigadores no publican, en tanto el 5\% que más artículos firma publica más de dos (Psicobiología; Psicología Evolutiva y de la Educación; y Psicología Social) o tres (Personalidad, Evaluación y Tratamiento Psicológico; y Metodología de las Ciencias del Comportamiento) artículos al año.

Eliminados los valores extremos y outliers y constatado el ajuste a la curva normal de la variable promedio de artículos publicados por autor en las áreas de conocimiento (ver asimetría y curtosis $<2$ en la Tabla 3) ejecutamos un ANOVA para la comparación de la media de artículos publicados por autor como variable dependiente y el área como factor de agrupamiento. Los resultados pusieron de manifiesto un efecto significativo del área de conocimiento en el número de artículos publicados, $\mathrm{F}(5,521)=4,08, \mathrm{p}<$ $, 001, \eta^{2}=, 038$. Los contrastes a posteriori, prueba T3 de Dunnett, F de Levene $(5,519)$ $=8,06, \mathrm{p}<, 001$, mostraron que los autores del área de Psicología de la Personalidad, Evaluación y Tratamiento Psicológico $(M=1,68)$ publican más artículos que los de las áreas de Psicología Evolutiva y de la Educación $(\mathrm{M}=1,22, \mathrm{~d}=0,46)$ y Psicología Básica $(\mathrm{M}=1,25, \mathrm{~d}=0,44)$. Dichas diferencias son de tal magnitud que superan al $66,93 \%$ de todas las posibles, entre las áreas de Personalidad, Evaluación y Tratamiento Psicológico y Psicología Evolutiva y de la Educación, y al 62,17\% entre las áreas de Personalidad, Evaluación y Tratamiento Psicológico y Psicología Básica. Es decir, no sólo hay diferencias significativas en la firma de artículos por autor entre estas áreas de conocimiento, sino que son muy notables.

\section{DISCUSIÓN}

La fuente de datos de este estudio tiene unas limitaciones que afectan a los resultados y que han de tenerse en cuenta a la hora de generalizar los mismos. Primera, los artículos registrados en la Core Collection de la Web of Science no están todos publicados en revistas indexadas en el Journal Citation Reports, que es el criterio último para la clasificación de las aportaciones como artículos publicados en revistas de reconocida valía (JCR) que se recoge como criterio orientador para la obtención de una evaluación positiva en las áreas de Psicología. Segunda, la lista de investigadores fue tomada de la Web of Science Production of Spanish Psychology por lo que no es completa y puede que esté sesgada positivamente en términos de producción al tratarse de un censo voluntario y público. Tercera, la adscripción por área de conocimiento se refiere al momento actual, pudiendo algunos investigadores haber cambiado de área de conocimiento a lo largo de su carrera académica. Cuarta, no todos los investigadores registrados son Personal Docente Universitario funcionario para los que se convocó originalmente la evaluación de la actividad investigadora (Real Decreto 1086/1989, de 28 de agosto, sobre retribuciones del profesorado universitario). No obstante, se ha ampliado a otras figuras y para aquellas que no lo estén en el presente momento (e.g., 
becarios) podrán posteriormente recuperar esos años de producción investigadora. Quinta, la producción científica se limita a publicaciones de artículos en revistas de reconocido prestigio, pero ésta abarca más medios de publicación (e.g., libros, capítulos de libros, artículos en revistas no indexadas en el JCR), por lo que las conclusiones sólo alcanzan a este tipo de publicaciones. Con estas consideraciones en mente, de los resultados de este estudio de archivo se deduce que:

1. Los niveles de producción por autor difieren, tanto en la producción media como en el número de valores atípicos, entre las áreas de conocimiento. En consecuencia, los criterios de clasificación y, por extensión, de calificación no tienen por qué ser generalizables inter-áreas de conocimiento.

2. Al igual que está recogido como criterio general para todos los campos que el solicitante deberá haber participado activamente en los trabajos que le dieron origen; concretamente, en los casos de multiautoría, los investigadores que se presenten a una evaluación de la actividad investigadora y que en el período que se va a evaluar, o en un año de dicho período, tengan una producción de artículos que entre en la región de valores atípicos en su área de conocimiento (promedio de artículos indexados en la Core Collection de la Web of Science en el período que se va a evaluar $>3,86$ en Psicobiología; > 5,57 en Personalidad, Evaluación y Tratamiento Psicológico; > 3,60 en Psicología Evolutiva y de la Educación; > 5,46 en Metodología de las Ciencias del Comportamiento; > 3,72 en Psicología Básica; y $>3,85$ en Psicología Social), han de justificar debidamente la participación en cada una de las aportaciones. La participación total en cada una de ellas resulta estadísticamente, y también en términos de tiempo, inasumible. De no justificarse suficientemente una participación en el total del artículo que se va a evaluar, procede la contemplada reducción de la calificación numérica asignada a la aportación.

3. Por el contario, al menos el $5 \%$ de los investigadores no publican en medios de reconocida valía, es decir, no cumplen con el deber científico (Artículo 40 del texto consolidado de la Ley Orgánica 6/2001, de 21 de diciembre, de Universidades que establece la investigación como un derecho y el deber del profesorado universitario).

4. El 25\% de los investigadores están justo en el límite (Psicobiología; Personalidad, Evaluación y Tratamiento Psicológico; y Metodología de las Ciencias del Comportamiento) o no llegan a producir 5 aportaciones indexadas en la Core Collection de la Web of Science en un tramo de evaluación estándar (sexenio). En suma, en torno a $1 / 4$ de los investigadores no estarían en condiciones de optar a una evaluación positiva de la actividad investigadora (para obtener una evaluación positiva deberán presentarse 5 aportaciones en el $\mathrm{CV}$ abreviado y 4 , al menos, en revistas indexadas en JCR, con 3 de ellas en los cuartiles 1 ó 2, lo que es prácticamente imposible de cumplir con sólo 4 ó 5 aportaciones en revistas indexadas en el JCR).

5. Los investigadores del área de Psicología de la Personalidad, Evaluación y Tratamiento publican más artículos, pero también firman con más autores (Arce, Selaya, Palmer y Sanmarco, 2019), es decir, no se trata únicamente de una mayor producción en sí, sino de una multiautoría mayor. Por el contrario, los investigadores de las áreas de Psicología Evolutiva y de la Educación, y Psicología 
Básica publican un promedio menor de artículos. En consecuencia, los investigadores de Psicología de la Personalidad, Evaluación y Tratamiento Psicológico disponen de más aportaciones para someter a evaluación, ligadas a multiautoría por lo que debe exigírsele (y verificar) la aportación a los artículos que se van a evaluar $y$, en el caso de no ser una aportación a la totalidad del artículo, aplicar la prevista reducción de la calificación asignada a la aportación.

6. La mitad de la población de investigadores (mediana) cuenta con un número suficiente de aportaciones por tramo de investigación (sexenio) para someter a evaluación (5 más un mínimo de 2 adicionales y hasta otras 5 adicionales en Psicobiología), es decir, están en condiciones de optar a una evaluación positiva.

7. El $25 \%$ de los investigadores (límite superior autoría probable) publican más de 10 artículos (hasta 15 en Metodología de las Ciencias del Comportamiento y Personalidad, Evaluación y Tratamiento Psicológico) por tramo de investigación con lo que duplican, cuando menos, el número de aportaciones para someter a evaluación.

8. El 5\% de los investigadores triplican o cuadriplican (Personalidad, Evaluación y Tratamiento Psicológico; Metodología de las Ciencias del Comportamiento) el número de publicaciones evaluables en el criterio 3.a de la convocatoria (artículos publicados en revistas de reconocida valía) y de las que el criterio orientador para la obtención de una evaluación positiva exige, al menos 4 , y 3 de ellas en los cuartiles 1 ó 2 . En suma, estos investigadores disponen de un gran número de artículos publicados que cumplen en criterio 3.a, esto es, artículos de, por extensión, reconocida valía. Sería una anomalía, para considerar, que no obtuvieran una evaluación positiva (esta contingencia se daría en el caso de que 3 de las aportaciones no fueran publicadas en revistas clasificadas en los cuartiles 1 ó 2).

La coautoría es necesaria y conveniente, pero la investigación futura debería establecer cuál es la carga de trabajo que un investigador puede llegar a desarrollar en términos de producción de artículos científicos publicados en revistas de reconocido prestigio. Resta también por abordar el debate no sólo de quién es autor, sino el orden de firma (Codina, 2019), cuyos usos oscilan desde el orden alfabético (sin utilidad para la evaluación) a otras aproximaciones como la sequence-determines-credit o la firstlats-authoremphasis (Tschamtke, Hochberg, Rand, Resch y Krauss, 2007), o cómo cuantificar la contribución de cada autor a la aportación.

\section{BIBLIOGRAFÍA}

Amado, B.G., Arce, R. y Herraiz, A. (2015). Psychological injury in victims of child sexual abuse: A meta-analytic review. Psychosocial Intervention, 24 (1), 49-62. https://doi.org/10.1016/j.psi.2015.03.002

Arce, R., Selaya, A., Palmer, A.L. y Sanmarco, J. (2019). En busca de una definición empírica de la multi-autoría desproporcionada para la evaluación de la actividad investigadora en Psicología, Revista de Investigación en Educación, 17(3), 196-207.

Codina, L. (2019). Artículos científicos: Quién puede firmarlos y en qué orden. Orden, ética y pragmatismo de la publicación académica, Revista ORL, 10 (3), 193-205. https://doi.org/10.14201/orl.19620

Fariña, F., Arce, R. y Novo, M. (2002). Heurístico de anclaje en las decisiones judiciales. Psicothema, 14, 39-46. Recuperado de: http://www.psicothema.com/pdf/684.pdf 
George, D. y Mallery, M. (2010). SPSS for windows step by step: A simple guide and reference, 17.0 update. Boston, MA: Pearson.

Ley Orgánica 6/2001, de 21 de diciembre, de Universidades, legislación consolidada (2006). Boletín Oficial del Estado, 307, 49400-49425 Recuperado de: https://www.boe.es/buscar/pdf/2001/BOE-A-2001-24515-consolidado.pdf

Monteiro, A., Vázquez, M.J., Seijo, D. y Arce, R. (2018). ¿Son los criterios de realidad válidos para clasificar y discernir entre memorias de hechos auto-experimentados y de eventos vistos en vídeo? Revista Iberoamericana de Psicología y Salud, 9 (2), 149-160. https://doi.org/10.23923/j.rips.2018.02.020

Orden de 2 de diciembre de 1994 por la que se establece el procedimiento para la evaluación de la actividad investigadora en desarrollo del Real Decreto 1086/1989, de 28 de agosto, sobre retribuciones del profesorado universitario. (1994). Boletín Oficial del Estado, 289, 3702837034. Recuperado de: https://www.boe.es/boe/dias/1994/12/03/pdfs/A37028-37034.pdf

Real Decreto 1086/1989, de 28 de agosto, sobre retribuciones del profesorado universitario. (1989). Boletín Oficial del Estado, 216, 28653-28656. Recuperado de: https://www.boe.es/boe/dias/1989/09/09/pdfs/A28653-28656.pdf

Resolución de 14 de noviembre de 2018, de la Comisión Nacional Evaluadora de la Actividad Investigadora, por la que se publican los criterios específicos aprobados para cada uno de los campos de evaluación. (2018). Boletín Oficial del Estado, 285, 115.199-115.222.

Recuperado de: https://www.boe.es/boe/dias/2018/11/26/pdfs/BOE-A-2018-16138.pdf

Tschamtke, T., Hochberg, M.E., Rand, T.A., Resh, V. y Krauss, J. (2007). Author sequence and credit for contributions in multiauthored publications. PLoS Biology 5 (1), e18. https://doi.org/10.1371/journal.pbio.0050018

Vilariño, M., Amado, B.G., Vázquez, M.J. y Arce, R. (2018). Psychological harm in women victims of intimate partner violence: Epidemiology and quantification of injury in mental health markers. Psychosocial Intervention, 27 (3), 145-152.

https://doi.org/10.5093/pi2018a23 